\title{
Diagnostic value of acustic radiation force impulse imaging in the assessment of salivary gland involvement in primary Sjögren's sydrome
}

\author{
Hale Turnaoglu', Feride Kural Rahatli', Melih Pamukcu², Kemal Murat Haberal', \\ Nihal Uslu ${ }^{1}$
}

${ }^{1}$ Department of Radiology, ${ }^{2}$ Department of Rheumatology, Başkent University, Faculty of Medicine, Çankaya, Ankara, Turkey

\begin{abstract}
Aims: The aim of this study is to investigate the diagnostic value of Acoustic Radiation Force Impulse (ARFI) imaging in the assessment of salivary gland involvement in primary Sjögren's syndrome (pSS). Material and methods: Twenty five patients with pSS and 25 healthy volunteers were included. First, echostructures and the thickness of the submandibular and parotid glands were evaluated by B-mode ultrasonography. Then, ARFI imaging with Virtual Touch Quantification ${ }^{\circledR}$ was performed. Ten independent shear wave velocity measurements were taken from each gland. Finally, the mean shear wave velocity (SWV) values were calculated, and used for further analysis. Results: The mean SWV values of parotid and submandibular glands were significantly higher in the pSS patients than in the healthy control group ( $\mathrm{p}<0.001)$. The cut-off of SWV values were calculated to be $1.98 \mathrm{~m} / \mathrm{s}$ for submandibular glands, and $1.93 \mathrm{~m} / \mathrm{s}$ for parotid glands. In pSS patients, the mean SWV values of parotid glands were higher than those of the submandibular glands $(p<0.001)$ and no statistically significant relationships between symptom duration or the degree of xerostomia and mean SWV values of parotid and submandibular glands were found (all $\mathrm{p}>0.005$ ). Conclusions: Our findings indicate that ARFI imaging may provide a non-invasive, simple and fast means of assessment of glandular impairment as an alternative test when other salivary gland tests are inconclusive or cannot be performed. ARFI may be a valuable adjunct for the clinical diagnosis of pSS.
\end{abstract}

Keywords: Sjögren's sydrome; salivary glands; ARFI; elastography

Primary Sjögren's syndrome (pSS) is a chronic inflammatory autoimmune disease that affects the salivary and lacrimal glands, resulting in xerostomia and xerophthalmia. Extraglandular manifestations in the joints, kidneys, respiratory tract, gastrointestinal tract and the vascular system may occur [1]. According to the latest recommendations issued by the American College of

Received 18.02.2018 Accepted 04.05.2018

Med Ultrason

2018, Vol. 20, No 3, 313-318

Corresponding author: Hale Turnaoglu, MD

Baskent University Faculty of Medicine, Ankara Hospital, Department of Radiology,

Mareşal Fevzi Çakmak Cad. 10. Sok. No: 45

06490 Bahcelievler, Ankara, Turkey

Phone: +90 5323106920

Fax: +90 3122237333

E-mail: haletrn@yahoo.com
Rheumatology (ACR) in 2016, diagnosis of pSS requires a total score of $\geq 4$ of the following 5 objective criteria, in patients with at least one symptom of ocular or oral dryness, or suspicion of pSS due to systemic features: (i) presence of focal lymphocytic sialadenitis with a focus score $>1$ focus $/ 4 \mathrm{~mm}^{2}$ in a labial salivary gland biopsy (score: 3); (ii) anti-SSA and/or anti-SSB positivity (score: 3 ); (iii) an abnormal ocular staining score of $\geq 5$ or a van Bijsterveld score of $\geq 4$ in at least one eye (score: 1); (iv) a Schirmer's test result of $\leq 5 \mathrm{~mm} / 5$ minutes in at least one eye (score: 1 ); (v) an unstimulated salivary flow rate of $\leq 0.1 \mathrm{ml} /$ minute (score: 1 ) [2].

Measuring of the unstimulated salivary flow rate, which is useful for the assessment of salivary gland involvement, is under critism because of its great variation over time in a single patient and its limited sensitivity and specificity [3]. On the other hand, a labial salivary 
gland biopsy, which is the most important test in the assessment of the salivary gland involvement, is invasive and, therefore, may cause complications. Furthermore, a lip biopsy can be misread by the pathologist. On a review of biopsies diagnosed as pSS, over half of the patients were reclassified [4]. There is a need for a reliable, noninvasive method for the assessment of salivary gland involvement.

Ultrasonography (US) is among the imaging techniques used to help diagnose pSS. The presence of parenchymal inhomogeneity (PIH) in pSS patients correlated well with the sialographic, scintigraphic and histological results. Nevertheless, a sonographically homogeneous parenchyma or mild PIH does not exclude pSS [5]. US elastography is a novel, non-invasive imaging method evaluating tissue stiffness. There are several types of elastographic examinations. Acoustic radiation force impulse (ARFI) imaging is an elastography technique that provides an objective numerical evaluation of tissue stiffness differently from the other elastography techniques [6]. It provides an estimate of tissue elasticity by measuring the propagation of shear waves emitted during induced tissue displacements [7]. The speed of the shear waves can be measured as shear wave velocity (SWV) and it is expressed quantitatively in meters per second $(\mathrm{m} / \mathrm{s})$. Stiffer tissues are associated with a higher SWV [8]. The benefits and usefulness of ARFI imaging have been shown in many clinical conditions, such as lesion detection and classification (e.g., in the breast, thyroid and prostate) and liver fibrosis staging [6,9-12]. There are also a few studies regarding the contribution and reliability of ARFI imaging to the diagnosis of pSS [7,1315]. The aim of this prospective study is to investigate the diagnostic value of ARFI imaging in the assessment of salivary gland involvement in pSS.

\section{Materials and methods}

\section{Study population}

Twenty five patients diagnosed with pSS according to ACR 2016 and 25 healthy volunteers were included in this study. This study was approved by the medical ethics committee of the Baskent University Ankara Hospital. Informed consent was obtained from all subjects, according to the World Medical Association Declaration of Helsinki, revised in 2000, Edinburgh. Exclusion criteria included: the presence of any other associated autoimmune disease, past operations affecting the parotid or submandibular gland, past head and neck radiation treatment, history of sialolithiasis and history of using anticholinergic drugs. Healthy volunteers with preexisting autoimmune disease, salivary gland-related disease or symptoms such as xerostomia, were exluded from the study. All patients' clinical records, including their age, sex, antibody positivities, minor salivary gland biopsy results, Schirmer's test results, and the date of onset of symptoms were noted.

\section{Imaging technique}

Ultrasonographic studies were performed using a 9-14-MHz linear transducer (Acuson S3000, Siemens Healthcare, Erlangen, Germany) and adequate software for performing elastographic examinations in quantitative ARFI mode (Virtual Touch Quantification ${ }^{\circledR}, \mathrm{VTQ}$ ). All patients were examined by the same operator. First, B-mode US was performed for all patients in order to assess glandular morphology. The echo structure of the parotid and submandibular glands in B-mode US was graded on a scale of 0 to 4 according to a previously published scoring system [5]: stage $0=$ normal homogeneity; stage $1=$ mild parenchymal inhomogeneity $(\mathrm{PIH})$ with hypoechoic areas $<2 \mathrm{~mm}$; stage $2=$ evident PIH with hypoechoic areas of 2-6 $\mathrm{mm}$; stage $3=$ gross $\mathrm{PIH}$ with hypoechoic areas $>6 \mathrm{~mm}$; and stage $4=$ adipose degeneration of the gland, adipose tissue echogenicity, and parenchymal atrophy. Also, the thickness of the parotid and submandibular glands were measured in the longitudinal plane. ARFI (VTQ) mode was then performed on the long-axis dimension of the parotid and submandibular glands. The transducer was gently applied together with a sufficient amount of contact gel. Every examination consisted of ten independent ARFI measurement (SWV) taken at a depth of approximately $1.0-1.5 \mathrm{~cm}$ in the center of the caudal pole of the parotid gland and the center of the submandibular gland, timed to the absence of swallowing maneuvers. The mean SWV value of the ten single SWV measurements was calculated for each gland, and used for further analysis.

\section{Statistical analysis}

All statistical analyses were performed using the Statistical Package for the Social Sciences (SPSS) software, Version 23.0 (SPSS, Chicago, IL, USA). Numerical variables, $\mathrm{n} \rightarrow \infty$ according to the Law of Large Numbers, the distribution of the sample mean approximates the normal distribution. Furthermore, since the numerical variables have a skewness (value / st.error) and a kurtosis (value / st.error) ratios of \pm 2 , the assumption is made that the normal distribution is appropriate. For numerical variables, mean, standard deviation, minimum and maximum values are given from the central tendency measures. Frequency distributions for categorical variables are given. An independent sample $t$ test was used to examine the difference between the two independent groups. A dependent sample $t$ test was used because the difference between submandibular glands and parotid 
glands was examined and groups were dependent. In addition, pearson correlation coefficients were used in examining the relationship between the duration of symptoms and ARFI values. Cut-off values for submandibular and parotid glands were calculated, for use in the detection of salivary gland involvement in pSS.

\section{Results}

The mean age of the patients with pSS was 51.6 years (range 21-77 years), and in the healthy control group 47.3 years (range 19-78 years). We detected a female predominance in pSS patients (24 females, 96\%). In the control group, there were 21 females (84\%). Clinical features of the pSS patients were summarized in table I. In the patient group, the mean duration of symptoms was 44.5 months (range 2 months-10 years). In the evaluation of the US

Table I. Clinical features of the primary Sjögren's syndrome patients $(n=25)$

\begin{tabular}{ll}
\hline Feature & Frequency (\%) \\
\hline Xerostomia & $22 / 25(88)$ \\
Xerophthalmia & $24 / 25(96)$ \\
Positive Schirmer's test & $22 / 25(88)$ \\
Positive biopsy results* & $12 / 25^{*}(48)$ \\
Autoantibodies positivity & \\
$\quad$ ANA & $21 / 25(84)$ \\
$\quad$ Anti-Ro & $9 / 25(36)$ \\
$\quad$ Anti-La & $4 / 25(16)$ \\
Rheumatoid factor & $6 / 25(24)$ \\
\hline
\end{tabular}

*Biopsy was not carried out in 8 (32\%) patients.
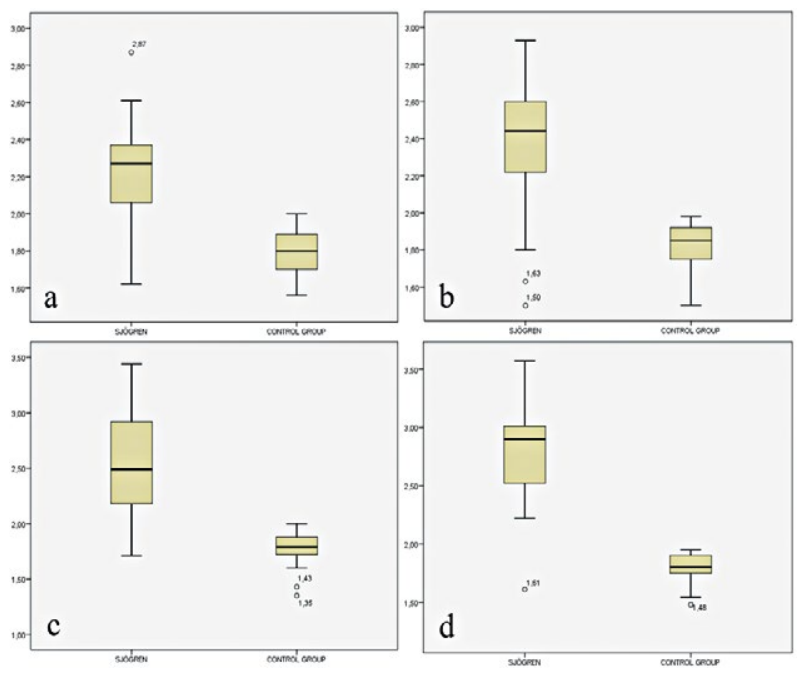

Fig 1. Box plots of mean right submandibular gland (a), left submandibular gland (b), right parotid gland (c), and left parotid gland (d) shear wave velocity (SWV) values in primary Sjögren's syndrome patients $(n=25)$ and in the control group $(n=25)$. Solid line: median SWV values in $\mathrm{m} / \mathrm{s}$. patterns of the parotid and submandibular glands, the findings were concordant bilaterally in all cases. Stage 0 (homogeneous gland parenchyma) was found in 17 (68\%) patients, stage 1 (mild inhomogeneity) in $6(24 \%)$ and stage 3 (gross inhomogeneity) in $2(8 \%)$ patients. In the control group, PIH was not observed. The values of mean thickness in the right submandibular, left submandibular, right parotid, and left parotid glands were calculated as $11.8 \pm 2.3 \mathrm{~mm}, 11.3 \pm 1.6,16.0 \pm 3.6,15.1 \pm 2.3 \mathrm{in}$ pSS patients, and $11.2 \pm 1.3,11.6 \pm 1.2,17.8 \pm 2.4,17.6 \pm 2.0$ in the control group, respectively. There were no statisti-

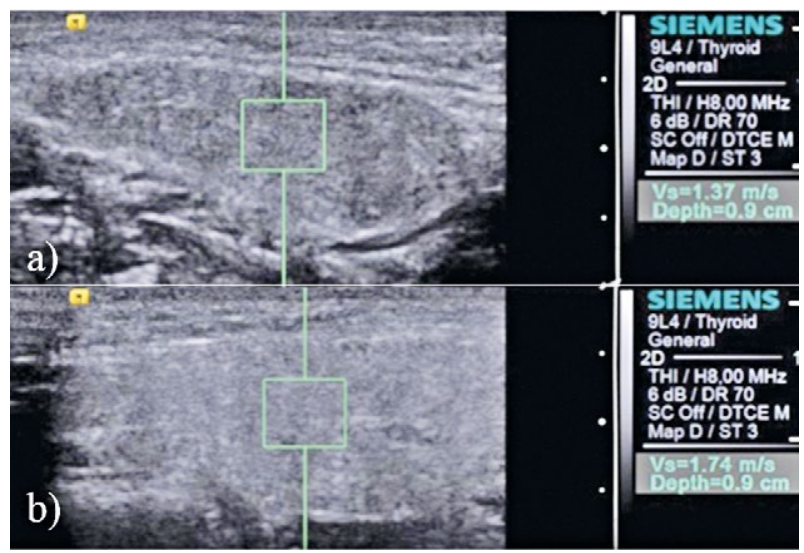

Fig 2. Shear wave velocity (SWV) measurements in the left submandibular (a) and left parotid glands (b) of a 33-year-old healthy control volunteer. The SWV of the glands were measured with fixed measurement sample dimensions of $5 \times 5 \mathrm{~mm}$. In this patient, the mean SWV of the left submandibular and parotid gland were calculated as $1.50 \mathrm{~m} / \mathrm{s}$, and $1.76 \mathrm{~m} / \mathrm{s}$ respectively.

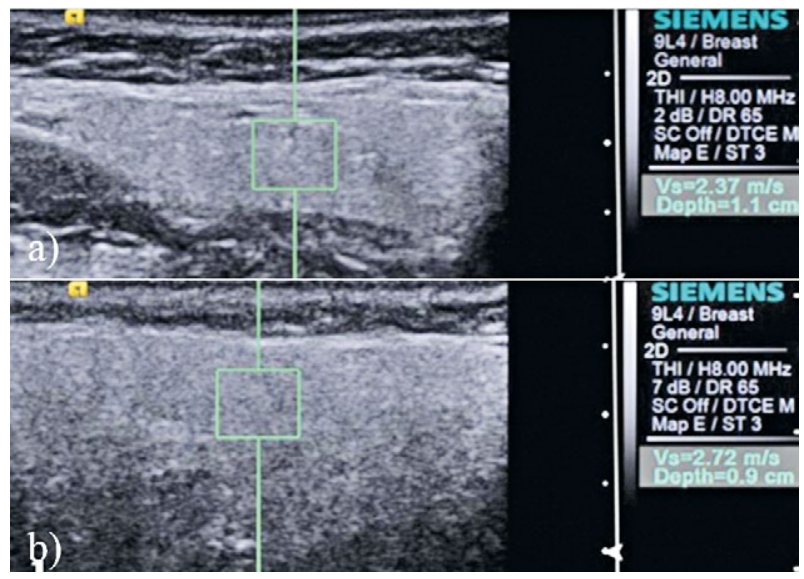

Fig 3. Shear wave velocity (SWV) measurements in the left submandibular (a) and left parotid glands (b) of a 58-year-old primary Sjögren's syndrome patient, who was diagnosed 2 years previous. The SWV of the glands were measured with fixed measurement sample dimensions of $5 \times 5 \mathrm{~mm}$. In this patient, the mean SWV of the left submandibular and parotid glands were calculated as $2.43 \mathrm{~m} / \mathrm{s}$, and $2.84 \mathrm{~m} / \mathrm{s}$ respectively. The glands' parenchyma are seen as homogeneous. 
Table II. The mean shear wave velocity values of salivary glands in primary Sjögren's syndrome patients and in the healthy control group

\begin{tabular}{lll}
\hline Salivary glands & Mean SWV in pSS patients (m/s) & Mean SWV in control group (m/s) \\
\hline Right submandibular gland & $2.20 \pm 0.28$ & $1.80 \pm 0.14$ \\
Left submandibular gland & $2.39 \pm 0.39$ & $1.81 \pm 0.14$ \\
Right parotid gland & $2.57 \pm 0.48$ & $1.78 \pm 0.15$ \\
Left parotid gland & $2.80 \pm 0.44$ & $1.79 \pm 0.13$ \\
\hline
\end{tabular}

SWV - shear wave velocity; pSS - primary Sjögren's syndrome

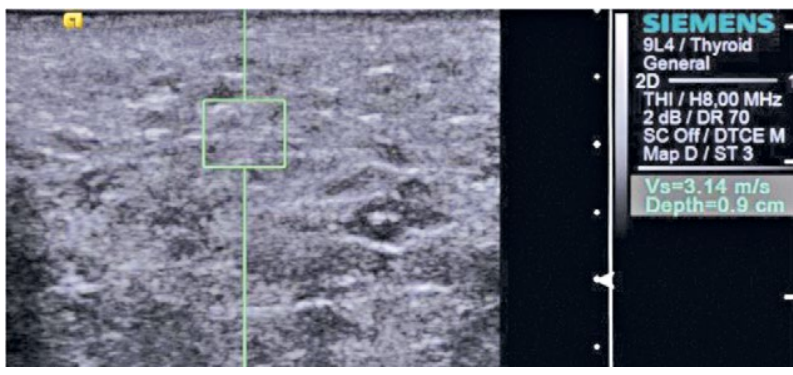

Fig 4. Shear wave velocity (SWV) measurement in the left parotid gland of a 49-year-old primary Sjögren's syndrome patient, who was diagnosed 7 years previous. In this patient, the mean SWV of the left parotid gland was calculated to be 2.91 $\mathrm{m} / \mathrm{s}$. Gross parenchymal inhomogeneity with hypoechoic areas are seen in the gland parenchyma.

cally significant differences in the thickness of salivary glands between the two groups.

The mean SWV values of parotid and submandibular glands were significantly higher in the pSS patients than in the healthy control group $(\mathrm{p}<0.001)$ (fig 1-4) (table II). The cut-off SWV values were calculated as $1.98 \mathrm{~m} / \mathrm{s}$ for submandibular glands (sensitivity 0.84 , specificity 0.92 ), and $1.93 \mathrm{~m} / \mathrm{s}$ for parotid glands (sensitivity 0.92 , specificity 0.92 ). As a result of the applied dependent sample $\mathrm{t}$ test, in pSS patients, the mean SWV values of parotid glands were found to be statistically significantly higher than the mean SWV values of submandibular glands $(\mathrm{p}<0.001)$.

In pSS patients, there was no statistically significant relationship between symptom duration or the degree of xerostomia and mean SWV values of parotid and submandibular glands ( $p>0.005)$.

\section{Discussions}

Our data indicate that ARFI imaging is a valuable tool in detecting salivary gland involvement in pSS. Recently, the effectiveness of ARFI imaging has been proven in detecting and assessing many conditions such as liver fibrosis or lesion characterization in many organs [6,912]. With respect to salivary glands, there are studies that demonstrated higher SWV values by ARFI imaging in salivary glands previously exposed to radiotherapy, with inflammatory sialadenitis and with known sialolithiasis, than in normal glands $[16,17]$. There are also a few published sources regarding the contribution of ARFI imaging in pSS [7,13-15]. In their study, Hofauer et al [14] indicated that the most promising results among the sonoelastographic modalities in pSS, as an examiner-independent modality for the evaluation of salivary gland stiffness, was the ARFI imaging. Knopf et al [13] concluded that ARFI imaging provided reliable, non-invasive assessment of glandular impairment for identifying early pSS stages and in addition to histology, it was the most important diagnostic tool for identifying early pSS. Samier-Guarin et al [7] indicated that the ARFI imaging may contribute to the diagnosis of pSS, as a non-invasive investigation that can demostrate abnormal architectural changes in the parotid glands. Zhang et al [15] concluded that ARFI imaging (VTQ and also VTIQ) might be a valuable adjunct to the gray-scale US for the clinical diagnosis of pSS. In our study, we also found that, by ARFI imaging, SWV values of the parotid and submandibular glands were significantly higher in patients with pSS than in the healthy control group, even in the early stages of the disease.

In our subjects, the mean SWV values of parotid glands were statistically significantly higher than the mean SWV values of submandibular glands in pSS patients. In the control group, there was relative symmetry of salivary gland elasticity and the difference was not statistically significant. Previously published studies are conflicting about this matter, which used different imaging modalities. Izumi et al [18] obtained data confirming the histological results reported by Block et al [19], by using computed tomography and magnetic resonance imaging. They indicated that the major salivary glands also contained fatty infiltrates in patients with pSS and the fat signal seemed similar in the parotid and submandibular glands, although no numerical comparison between the two was reported. Zhang et al [15] reported that the stiffness of parotid glands were not significantly different than those of the submandibular gland by ARFI imaging. However, the sialography results reported by Golder et al [20] are consistent with our results: they indicated that the lymphocytic infiltrate was more severe in the parotid 
glands than in the submandibular glands in patients with pSS. Also, in their studies, Samier Guarin et al [7], Knopf et al. [13], and Hofauer at al. [14] reported a discrepancy in SWV values between the parotid and submandibular glands, by ARFI imaging, similar to our results. This discrepancy is most likely related to the different histological composition of the parotid and submandibular glands [13]. While the serous parotid (and lacrimal) gland is primarily affected by pSS, the mixed submandibular (and sublingual) gland is impaired to a lesser extent [21]. The exiguity of histological data on the parotid glands with pSS is probably attributable to the fact that parotid gland biopsies are not performed in this indication, given the risk of injury to blood vessels and nerves [13]. However, it may suggest that the histological changes, as lymphocytic sialadenitis, and the development of fibrosis might be more marked and/or occur earlier in the parotid glands than in the submandibular glands. It may also suggest that the evaluation of parotid glands may contribute to the diagnosis of pSS-in the early stages of the disease, while the minor salivary gland biopsy is negative.

Our analysis of side-to-side correlations for parotid and submandibular gland SWV values indicated a symmetrical distribution of the elasticity changes. However, Samier-Guerin et al [7] reported an asymmetrical distribution in parotid and submandibular gland SWV values. Also, Golder et al [20] and Güne et al [22], reported in their studies that the inflammation of the major salivary glands in pSS is asymmetrical, at least in the early stages of the disease. Our findings suggest that a mean parotid gland SWV value greater than $1.93 \mathrm{~m} / \mathrm{s}$ and a mean submandibular gland SWV value greater than $1.98 \mathrm{~m} / \mathrm{s}$ may constitute an indirect indicator of structural changes in the salivary gland parenchyma. Nevertheless, the individual values showed little overlap between the pSS patients and the control group. Probably, this condition is, again, due to the asymmetrical involvement of the gland parenchyma. Therefore, both sides of the salivary glands must be examined and the mean SWV value of ten independent measurements should be used for each gland.

Several studies suggest that US may contribute to the diagnosis of pSS [23-25]. Patients with pSS may have an inhomogeneous salivary-gland structure due to the gradual development of hypoechoic foci. Nevertheless, a sonographically homogeneous parenchyma or mild heterogeneity does not exclude pSS [5]. In support of this condition, $68 \%$ of pSS patients' glands parenchyma were homogeneous in our subjects. There are also studies about the role of real-time (strain) elastography in pSS patients $[26,27]$. In this technique, continuous transducer compression or external mechanical compression is required to induce strain on tissues. Real-time elastography is eas- ily influenced by several factors, such as operator dependency, poor reproducibility and non-quantitative results [16] and may be ineffective in the diagnosis of the early stages of pSS [26]. ARFI imaging is an elastography technique, which requires no external compression, and SWV values provide a quantitative evaluation of tissue stiffness. Knopf et al [13] reported that a reliable inter- and intra-rater reliability could be achieved with the implementation of a standard operating procedure. However, in their study Hofauer et al indicated that attention must be paid to ARFI imaging of the parotid gland, as inter-rater agreement between the experienced and inexperienced examiners was poor. Therefore, they recommended that only experienced examiners should performed ARFI imaging on parotid glands. Also they concluded that ARFI imaging in submandibular glands did not seem to be reproducible for both experienced and inexperienced examiners. Pre-compression of the tissue, swallowing, or speaking by the patient can influence the SWV values in all organs [14,28,29]. Application of different amounts of pressure by the probe during the examination, can lead to the different results [14]. During the measurements of SWV it is necessary to pay attention to these points. In our study, all measurements were done by the same operator with 5 years experience in ARFI imaging and great care was taken not to compress the glands. Currently, only the most sophisticated ultrasound machines are equipped with ARFI imaging software but ARFI imaging software will become increasingly available [7].

In our study, the age distribution of pSS patients and the control group was similar. Samier-Guerin et al already reported that there is no evidence that parotid or submandibular gland SWV values correlated with age in healthy controls aged 18 to 60 years [7].

The small sample size is the major limitation of our study. Also female dominancy may be considered as a limitation. Further evaluations are necessary, in a larger sample, in order to determine whether age is a potential confounding factor and to establish the accurate cut-off values.

In conclusion our findings indicate that ARFI imaging is a non-invasive, simple and fast imaging technique for assessment the salivary gland parenchyma. The method can be used as an alternative test in the cases with inconclusive classical tests and may be a valuable adjunct for the clinical diagnosis of pSS.

\section{References}

1. Moutsopoulos HM. Sjögren's syndrome. In: Schumacher HR (ed). Primer on the rheumatic diseases, 10th Edition, Atlanta, GA: Arthritis Foundation, 1993:131-135. 
2. Shiboski CH, Shiboski SC, Seror R, et al; International Sjögren's Syndrome Criteria Working Group. 2016 American College of Rheumatology/ European League Against Rheumatism Classification Criteria for Primary Sjögren's Syndrome: A Consensus and Data-Driven Methodology Involving Three International Patient Cohorts. Arthritis Rheumatol 2017;69:35-45.

3. Speight PM, Kaul A, Melsom RD. Measurement of whole unstimulated salivary flow in the diagnosis of Sjögren's syndrome. Ann Rheum Dis 1992;51:499-502.

4. Vivino FB, Gala I, Hermann GA. Change in final diagnosis on second evaluation of labial minor salivary gland biopsies. J Rheumatol 2002;29:938-944.

5. Makula E, Pokorny G, Rajtar M, Kiss I, Kovacs A, Kovacs L. Parotid gland ultrasonography as a diagnostic tool in Primary sjögren's syndrome. Br J Rheumatol 1996;35:972977.

6. Sporea I, Sirli R, Popescu A, Danila M. Acoustic Radiation Force Impulse (ARFI)-a new modality for the evaluation of liver fibrosis. Med Ultrason 2010;12:26-31.

7. Samier-Guérin A, Saraux A, Gestin S, et al. Can ARFI elastometry of the salivary glands contribute to the diagnosis of Sjögren's syndrome? Joint Bone Spine 2016;83:301-306.

8. Matsuzuka T, Suzuki M, Saijo S, et al. Stiffness of salivary gland and tumor measured by new ultrasonic techniques: Virtual touch quantification and IQ. Auris Nasus Larynx 2015;42:128-133.

9. Barr RG. Elastography in clinical practice. Radiol Clin North Am 2014;52:1145-1162.

10. D'Onofrio M, Crosara S, De Robertis R, et al. Acoustic radiation force impulse of the liver. World J Gastroenterol 2013;19:4841-4849.

11. Sporea I, Sirli R, Bota S, Vlad M, Popescu A, Zosin I. ARFI elastography for the evaluation of diffuse thyroid gland pathology: Preliminary results. World J Radiol 2012;4:174-178.

12. Tozaki M, Isobe S, Sakamoto M. Combination of elastography and tissue quantification using the acoustic radiation force impulse (ARFI) technology for differential diagnosis of breast masses. Jpn J Radiol 2012;30:659-670.

13. Knopf A, Hofauer B, Thürmel K, et al. Diagnostic utility of Acoustic Radiation Force Impulse (ARFI) imaging in primary Sjoegren`s syndrome. Eur Radiol 2015;25:30273034.

14. Hofauer B, Mansour N, Heiser C, et al. Sonoelastographic Modalities in the Evaluation of Salivary Gland Characteristics in Sjögren's Syndrome. Ultrasound Med Biol 2016;42:2130-2139.

15. Zhang S, Zhu J, Zhang X, He J, Li J. Assessment of the Stiffness of Major Salivary Glands in Primary Sjögren's Syndrome through Quantitative Acoustic Radiation Force Impulse Imaging. Ultrasound Med Biol 2016;42:645-653.

16. Badea AF, Tamas Szora A, Ciuleanu E, et al. ARFI quantitative elastography of the submandibular glands. Normal measurements and the diagnosis value of the method in radiation submaxillitis. Med Ultrason 2013;5:173-179.

17. Zengel, P, Schrötzlmair F, Schwarz F, et al. Elastography: A new diagnostic tool for evaluation of obstructive diseases of the salivary glands; primary results. Clin Hemorheol Microcirc 2012;50:91-99.

18. Izumi M, Eguchi K, Nakamura H, Nagataki S, Nakamura T. Premature fat deposition in the salivary glands associated with Sjögren's syndrome: MR and CT evidence. AJNR Am J Neuroradiol 1997;18:951-958.

19. Bloch KJ, Buchanan WW, Wohl MJ, Bunim JJ. Sjögren's syndrome. A clinical, pathological, and serological study of sixty-two cases. Medicine 1992;71:386-401.

20. Golder W, Stiller M. Distribution pattern of Sjögren's syndrome: a sialographical study. Z Rheumatol 2014;73:928933.

21. Dankof A, Morawietz L, Feist E. Labial salivary gland biopsy in Sjogren's syndrome. Pathologe 2006;6:416-421.

22. Güne SE, Yilmaz S, Karalezli A, Aktas A. Quantitative and visual evaluation of salivary and thyroid glands in patients with primary Sjögren's syndrome using salivary gland scintigraphy: relationship with clinicopathological features of salivary, lacrimal and thyroid glands. Nucl Med Commun 2010;31:666-672.

23. Carotti M, Ciapetti A, Jousse-Joulin S, Salaffi F. Ultrasonography of the salivary glands: the role of grey-scale and colour/power Doppler. Clin Exp Rheumatol 2014;32:S61-S70.

24. Cornec D, Jousse-Joulin S, Marhadour T, et al. Salivary gland ultrasonography improves the diagnostic performance of the 2012 American College of Rheumatology classification criteria for Sjogren's syndrome. Rheumatology 2014;53:1604-1607.

25. Cornec D, Jousse-Joulin S, Pers JO, et al. Contribution of salivary gland ultrasonography to the diagnosis of Sjögren's syndrome: toward new diagnostic criteria? Arthritis Rheum 2013;65:216-225.

26. Gunes Tatar I, Altunoglu H, Kurt A, et al. The role of salivary gland elastosonography in Sjögren's syndrome: preliminary results. Int J Rheum Dis 2014;17:904-909.

27. Dejaco C, De Zordo T, Heber D, et al. Real-time sonoelastography of salivary glands for diagnosis and functional assessment of primary Sjögren's syndrome. Ultrasound Med Biol 2014;40:2759-2767.

28. Mantsopoulos K, Klintworth N, Iro H, Bozzato A. Applicability of shear wave elastography of the major salivary glands: values in healthy patients and effects of gender, smoking and pre-compression. Ultrasound Med Biol 2015;41:2310-2318.

29. Wojcinski S, Brandhorst K, Sadigh G, Hillemanns P, Degenhardt $F$. Acoustic radiation force impulse imaging with virtual touch tissue quantification: measurements of normal breast tissue and dependence on the degree of pre-compression. Ultrasound Med Biol 2013;39:2226-2232. 